\title{
Water Vapor-Plasma-Enhanced Oxidation of Thin Titanium Films
}

\author{
L. Pranevicius ${ }^{a, *}$, S. Tuckute ${ }^{a, b}$, L.L. Pranevicius ${ }^{a, b}$ And K. Gedvilas ${ }^{a}$ \\ ${ }^{a}$ Department of Physics, Vytautas Magnus University, 8 Vileikos St., LT-44404 Kaunas, Lithuania \\ ${ }^{b}$ Lithuanian Energy Institute, 3 Breslaujos St., LT-44403 Kaunas, Lithuania
}

\begin{abstract}
It is shown that the water vapor plasma processing offers modified Ti oxidation thermodynamics and kinetics over conventional, thermal oxidation. The $0.3-0.6 \mu \mathrm{m}$ thick Ti films were sputter-deposited on silicon substrates and subsequently treated with low-pressure water vapor plasma at room temperature under continuous injection of water cloud vapor into the vacuum chamber from the heated water container. The changes of microstructure, phase composition, elemental composition and surface morphology upon the RF-power dissipated in plasma and treatment duration were investigated. We conclude that oxygen diffusion is enhanced in the presence of water vapor plasma, and deduce that fast $\mathrm{H}^{+}$transients because of their high mobility may be responsible for oxygen diffusion enhancement. This phenomenon can be explained as the result of two coexisting and competing reactions of oxidation and reduction on the surface. The different plasma reduction/oxidation state on the surface can be maintained by coordinated adjustment of an intensity of plasma radiation. Analysis of the experimental results is used to obtain important insights into the behavior of water molecules adsorbed on the oxidized titanium surfaces exposed to water vapor plasma at room temperature.
\end{abstract}

DOI: 10.12693 /APhysPolA.123.907

PACS: 81.15.Cd, 68.47.Gh, 81.65.Mq, 66.30.jj

\section{Introduction}

Titanium dioxide $\left(\mathrm{TiO}_{2}\right)$ is the most widely used photocatalyst material for the variety of applications $[1,2]$. This is because it is photostable, chemically stable, photoactive, relatively inexpensive and non-toxic. However, the titanium dioxide absorbs only ultraviolet light and is transparent for visible light. Since the Honda-Fujishima effect was reported $[3,4]$ different approaches, including the use of dopants such as hydrogen $[5,6]$, have been taken to narrow the band gap of $\mathrm{TiO}_{2}$. It has been shown that hydrogenation changes the electronic properties of titanium dioxide powder by introducing a specific kind of disorder, mid-gap electronic states and reduces the band gap. This makes it possible for the visible light solar spectrum to be absorbed and contribute to the photocatalysis.

Titanium is slow to react with water and air at room temperature because it forms a protective oxide 1-2 nm thick barrier film reaching a thickness of $25 \mathrm{~nm}$ in four years that protects it from further reaction. It readily reacts with oxygen at $1200^{\circ} \mathrm{C}$ in air, and at $610^{\circ} \mathrm{C}$ in pure oxygen, forming $\mathrm{TiO}_{2}[7]$. It is known [8] that the enhanced oxidation of titanium in the presence of water vapor is caused by rapid matter transport through hydroxide ion defects and has to be related to the high reactivity of oxygen arising from water vapor.

The oxidation of titanium is strongly affected by the presence of plasma [9]. Low and atmospheric plasmas have been used. Experimentally it is shown that plasma

*corresponding author; e-mail: 1.pranevicius@gmf .vdu. It irradiation modifies surface properties in such a way that the predictions following classical thermodynamics become questionable. The outermost layer becomes hydrophilic [10], highly defected and new pathways for the transport of water molecules and hydrogen atoms become possible. The ion irradiation not only directly fills hydrogen into the subsurface region of the Ti film but also induces displacements of surface atoms, defects and disorders in the implantation region. Possible oxidation-reduction reactions of partially oxidized titanium by hydrogen plasma have been considered in publication [11]. Recently, the black visible light absorbing the double-layered hydrogenated $\mathrm{TiO}_{2} / \mathrm{Ti}$ thin films were fabricated by water vapor plasma at room temperature [12].

The complete explanation of the plasma activated processes during titanium oxidation using water vapor plasma has not been given yet. Plasma induced changes might consist of microstructural changes in the scale, enhancement or decrease in the oxidation rate, changes in transport processes through the oxide or alteration in the oxide spalling resistance. The role of hydrogen on the titania surfaces is still somewhat unclear [13]. There has been no research into what is the role of hydrogen, which is the product from the oxidation reaction, in the mechanism of oxidation.

The aim of this work was to elucidate the effects of the plasma activated processes and to acquire deeper understanding of the role of hydrogen in the mechanism of titanium oxidation by water vapor plasma.

\section{Experimental}

In this work we used the PVD-75 magnetron sputtering system manufactured by Kurt J. Lesker Co. with two independent Torus DC powered magnetrons. The system 
was equipped with a cryopump which allows to achieve a base vacuum level down to $5 \times 10^{-6} \mathrm{~Pa}$. For sputter deposition of thin titanium films we used $99.99 \%$ purity titanium targets obtained from Kurt J. Lesker Co. Prior to the deposition, the target was well cleaned in order to remove the surface oxide layers. Titanium films were deposited on the Si (111) $10 \times 20 \mathrm{~mm}^{2}$ surface. The sputtering time was chosen to obtain Ti films of the thickness in the range of 300-600 $\mathrm{nm}$ and the sputtering power was about $100 \mathrm{~W}$, that is corresponding to a power density of $1.2 \mathrm{~W} / \mathrm{cm}^{2}$. The film thickness has been evaluated by the stylus nanoprofilometer with an accuracy of about $10 \mathrm{~nm}$. The sample surface was about $200 \mathrm{~mm}^{2}$.

After the initial characterisation, the samples were moved into the vacuum 801 volume stainless steel chamber which was equipped with an inlet valve permitting controllable injection of water vapor into the chamber. The Ti samples to be treated were located on the water-cooled substrate holder. The reaction chamber was evacuated using a turbomolecular vacuum pump down to a pressure of about $10^{-3} \mathrm{~Pa}$. Once the steady state partial pressure of water vapor of $5-10 \mathrm{~Pa}$ in the chamber at ambient temperature was reached, the plasma generator was activated at a power of 50-300 W. With an axial magnetic field, the ionization degree of working gas approached the maximum value at about $300 \mathrm{~W}$ and then saturated. The densities of positive ions and electrons and the electron temperature were of around $5 \times 10^{10} \mathrm{~cm}^{-3}$ and between 1.2 and $1.6 \mathrm{eV}$, respectively, measured by the Langmuir probe at the processing power of $300 \mathrm{~W}$.

The microstructure of the as-deposited and plasma treated samples was characterized by the X-ray diffraction (XRD) method using the Bruker diffractometer (Bruker D8). The measurements were performed with $2 \theta$ angle in the range $20^{\circ}-70^{\circ}$ using $\mathrm{Cu} K_{\alpha}$ radiation in the steps of $0.01^{\circ}$. All X-ray diffraction peaks were indexed using software together with Search-Match function and PDF-2 database from the International Centre for Diffraction Data (ICDD). The thickness and surface topography of $\mathrm{Ti}$ films were measured using the stylus profiler (AMBIOS XP 200). The spatial resolution was several nanometers. The surface views before and after plasma treatment were investigated by the scanning electron (SEM, JEOL JSM - 5600) and optical (Nikon Eclipse LV150) microscopes. The distribution profiles of $\mathrm{O}$ atoms in the $\mathrm{Ti}$ films were measured by the Auger electron spectroscopy (AES, PHI 700XI). Complementary, the distribution profiles of $\mathrm{H}$ and $\mathrm{O}$ atoms in the plasma treated Ti films were measured by glow discharge optical emission spectroscopy (GDOES, Spectruma Analytic $\mathrm{Gmbh}$ ). The elemental composition of the plasma treated films was analyzed by energy dispersive X-ray spectroscopy (EDS, Bruker Quad 5040).

\section{Experimental results}

The Auger oxygen depth profile (Fig. 1, curve 2) shows sharp decrease of oxygen concentration from 75 at. $\%$ on the surface followed by gradual decrease through the entire film thickness in the bulk to the values around the limit solubility of oxygen in titanium (32 at.\%).

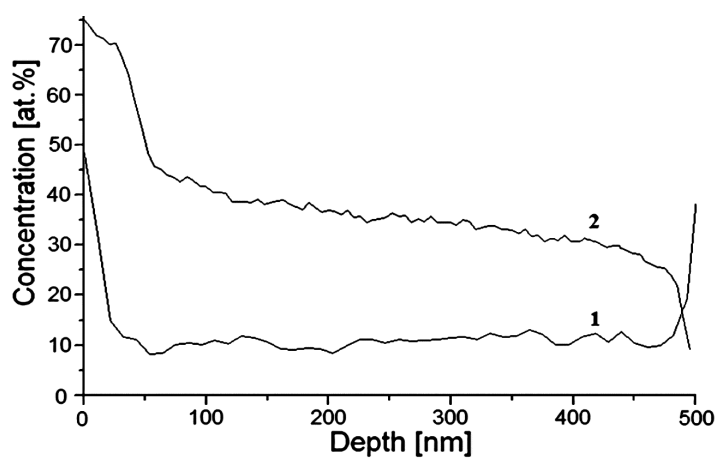

Fig. 1. The depth distribution profiles of $\mathrm{O}$ atoms in the Ti film: 1 - as-deposited, and 2 - plasma treated at $200 \mathrm{~W}$ for $5 \mathrm{~min}$.

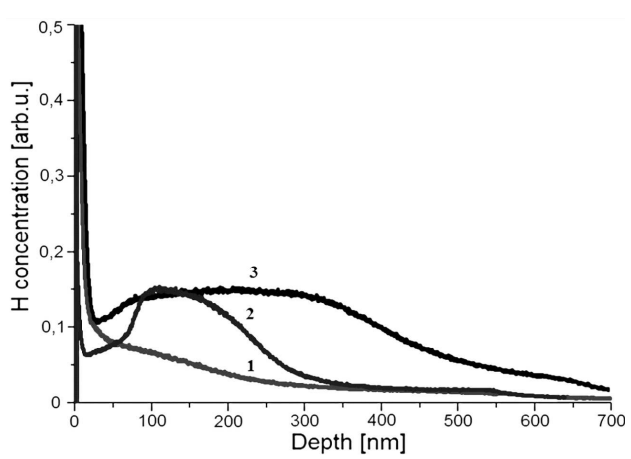

Fig. 2. GDOES depth distribution profiles of $\mathrm{H}$ atoms in the plasma treated $\mathrm{Ti}$ films for the ion current densities: $1-1 \mu \mathrm{A} / \mathrm{cm}^{2}, 2-50 \mu \mathrm{A} / \mathrm{cm}^{2}$, and $3-$ $500 \mu \mathrm{A} / \mathrm{cm}^{2}$.

Figure 2 presents the GDOES depth distribution profiles of $\mathrm{H}$ atoms in the plasma treated Ti films for different ion current densities extracted from the water vapor plasma and directed to the Ti film by the negative $200 \mathrm{~V}$ bias voltage: $1-1 \mu \mathrm{A} / \mathrm{cm}^{2}, 2-50 \mu \mathrm{A} / \mathrm{cm}^{2}$, and $3-$ $500 \mu \mathrm{A} / \mathrm{cm}^{2}$. It can be seen that the penetration depth of $\mathrm{H}$ atoms increases with the increasing ion irradiation intensity. The $20-30 \mathrm{~nm}$ thick surface layer is enriched by hydrogen atoms. It is also noticeable that the bulk concentration of hydrogen does not depend on the irradiation intensity and saturates as it reaches the solubility limit of 0.14 at.\% [14].

Figure 3 presents the XRD patterns of Ti films: 1 as-deposited, 2 - plasma treated for $5 \mathrm{~min}$ at $200 \mathrm{~W}$, and 3 - for $5 \mathrm{~min}$ at $300 \mathrm{~W}$. The XRD patterns show that the as-deposited amorphous (Fig. 3, curve 1) Ti film after being treated with water vapor plasma for $5 \mathrm{~min}$ at $200 \mathrm{~W}$ includes small peaks corresponding to crystalline $\mathrm{TiO}_{2}$ (Fig. 3, curve 2). Diffraction peaks characteristic of $\mathrm{TiO}_{2}$ and small peaks, which may be attributed to TiO, 


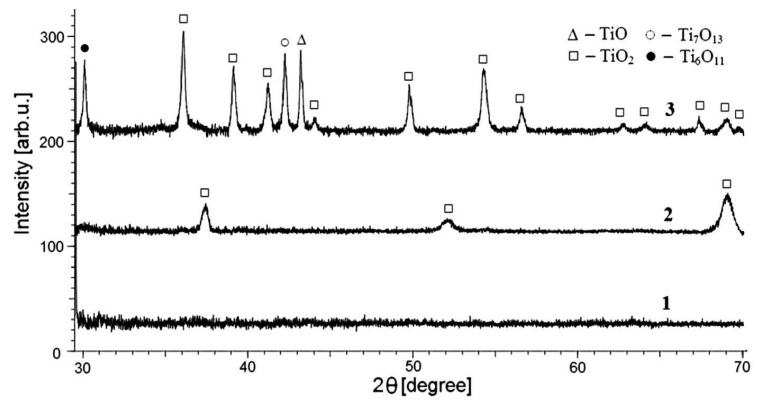

Fig. 3. XRD patterns of the Ti films: 1 - as-deposited, 2 - plasma treated for $5 \mathrm{~min}$ at $200 \mathrm{~W}$, and 3 - for $5 \mathrm{~min}$ at $300 \mathrm{~W}$.

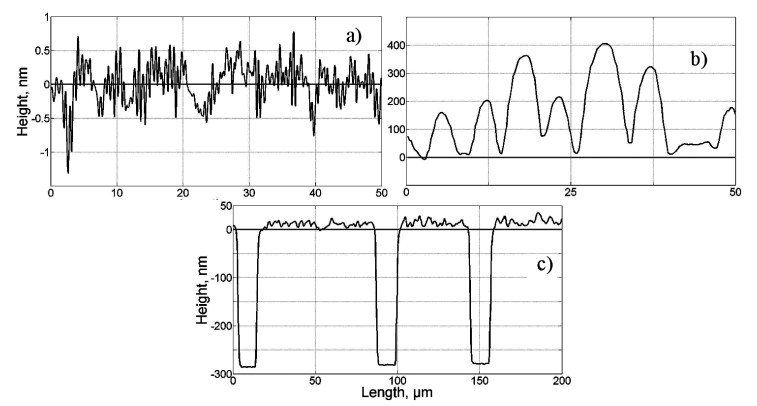

Fig. 4. Surface height profiles of the Ti films: (a) as-deposited, (b) plasma treated for 5 min at $200 \mathrm{~W}$, and (c) for 5 min at $300 \mathrm{~W}$.

$\mathrm{Ti}_{7} \mathrm{O}_{13}$ and $\mathrm{Ti}_{6} \mathrm{O}_{11}$, appear after treatment for 5 min at $300 \mathrm{~W}$ (Fig. 3, curve 3).

Figure 4 illustrates the surface height profiles of as-deposited, plasma treated for $5 \mathrm{~min}$ at $200 \mathrm{~W}$ and for $5 \mathrm{~min}$ at $300 \mathrm{~W}$ (Fig. $4 \mathrm{a}-\mathrm{c}$, respectively) Ti films. As follows the surface root-mean-square roughness of the as-deposited Ti film is less than $1 \mathrm{~nm}$ (Fig. 4a). The surface height topography is non-monotonic with the maxima at heights in the range of $200-400 \mathrm{~nm}$ above the reference level after plasma treatment for $5 \mathrm{~min}$ at $200 \mathrm{~W}$ (Fig. 4b).

The SEM surface view (Fig. 5a) reveals that these local height elevations are formed by randomly distributed 10$20 \mu \mathrm{m}$ circular bumps. Presumably, circular bumps are caused by hydrogen bubbles in the film. The increase of RF generator power from $200 \mathrm{~W}$ to $300 \mathrm{~W}$ changes surface topography of the plasma treated Ti film. The surface height profile (Fig. 4c) discloses tiny holes in the plasma treated film. The SEM surface view (Fig. 5b) reveals the randomly distributed circular bumps and holes on the surface of the Ti film treated for 5 min at $300 \mathrm{~W}$. It can be generalized that the experimental information supplied by these tests is related to the typical buckling and spalling of an oxide scale, which is attributed to the weak oxide-substrate interface under lateral compression. The buckle develops a normal tensile stress at the perimeter of the defect to produce fracture in the surface oxide scale.

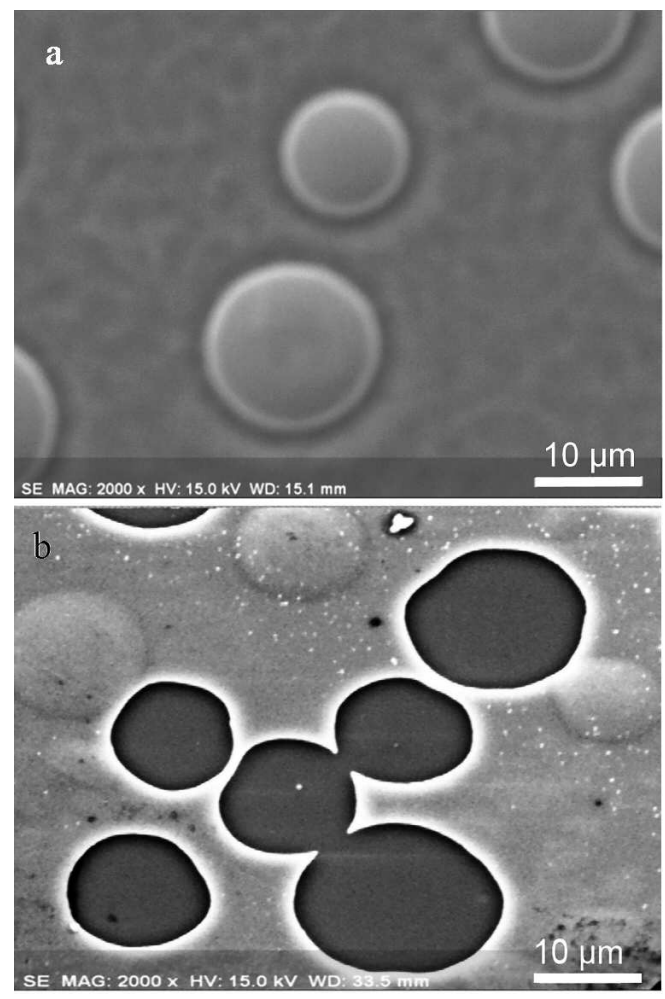

Fig. 5. SEM surface views of the plasma treated $\mathrm{Ti}$ films: (a) plasma treated for $5 \mathrm{~min}$ at $200 \mathrm{~W}$ and (b) for $5 \mathrm{~min}$ at $300 \mathrm{~W}$.

\section{Results and discussions}

Under the water vapor plasma treatment surface of $\mathrm{Ti}$ film is partially or entirely covered by adsorbed with the water layer. Adsorbed water molecules driven by superwettability effects on the hydrophilic surface of oxidized titanium converge to water island layers. The competing oxidation and reduction reactions occur only at the very top surface of titanium. Under these conditions, the kinetics of redox processes and the concentration of hydroxyl groups on the surface depend on the oxidation state of titanium oxide which is modified by the RF power dissipated in plasma.

Water molecules are split on the surface of plasma reduced $\mathrm{TiO}_{2}$ into $\mathrm{O}$ and $\mathrm{H}$ atoms. Oxygen atoms move through the uppermost $\mathrm{TiO}_{2}$ layer and reach the metallic $\mathrm{Ti}$ which is oxidized. Hydrogen ions are transported through the oxide layer governed by the oxidation rate. Subsequently, they recombine at the interface resulting in the formation of $\mathrm{H}_{2}$, as shown schematically in Fig. 6 . In this way, the plasma induced $\mathrm{TiO}_{2}$ reduction process enhances the chemical activity of surface, dissociative adsorption of water molecules and oxygen diffusion.

The electrochemical processes explaining the enhanced oxidation of the titanium film by water vapor plasma may be interpreted in the following way. Electrons and holes produced by plasma radiation in the oxidized layer are trapped by the surface $\mathrm{Ti}^{4+}$ ions and bridging site $\mathrm{O}^{2-}$ 


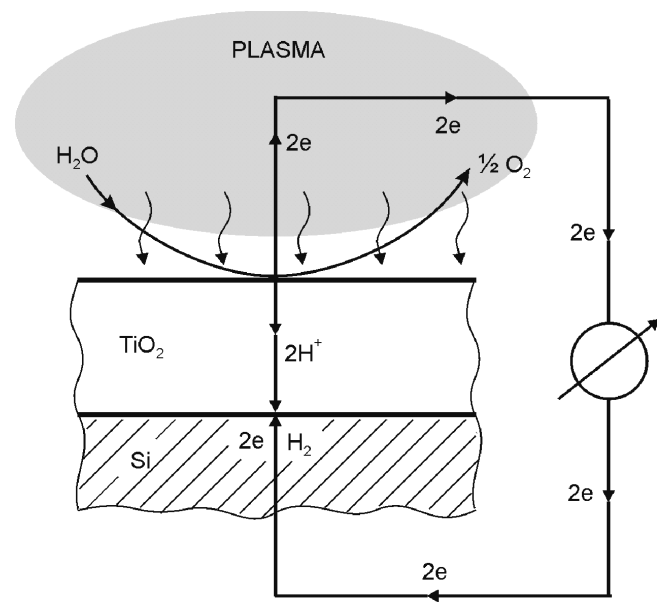

Fig. 6. Schematic representation of the mechanism of $\mathrm{H}^{+}$transfer.

ions to form $\mathrm{Ti}^{3+}$ ions and oxygen defects, where water molecules are dissociatively adsorbed resulting in the hydrophilic surface. In this process electroactive $\mathrm{Ti}^{3+}$ ions can be reoxidized to $\mathrm{Ti}^{4+}$ ions by anodic polarization. The set of possible reactions is: (i) $\mathrm{TiO}_{2}+h \nu \rightarrow$ $\mathrm{e}^{-}\left(\mathrm{TiO}_{2}\right)+h^{+}\left(\mathrm{TiO}_{2}\right)$, (ii) $h^{+}\left(\mathrm{TiO}_{2}\right)+\mathrm{H}_{2} \mathrm{O} \rightarrow 2 \mathrm{H}^{+}+$ $(1 / 2) \mathrm{O}_{2},(\mathrm{iii}) \mathrm{e}^{-}\left(\mathrm{TiO}_{2}\right)+4 \mathrm{H}^{+}+\mathrm{TiO}_{2} \rightarrow \mathrm{Ti}^{3+}+2 \mathrm{H}_{2} \mathrm{O}$, and (iv) $\mathrm{Ti}^{3+}+2 \mathrm{H}_{2} \mathrm{O} \rightarrow \mathrm{TiO}_{2}+4 \mathrm{H}^{+}+\mathrm{e}^{-}$.

We suppose that the fast $\mathrm{H}^{+}$transients, because of their high mobility, can be responsible for oxygen transport enhancement. The transfered $\mathrm{H}^{+}$recombines with electrons at the $\mathrm{TiO}_{2}$ interface boundary forming $\mathrm{H}_{2}$ molecules and bubbles. These factors cause that the oxidation rate of the areas covered by layers of adsorbed water molecules on the surface of the titanium film immersed into the water layer plasma significantly increases and is predominantly determined by the plasma radiation intensity.

\section{Conclusions}

An attempt to explain the enhanced oxidation of thin Ti films by water vapor plasma is made. As follows from our results, the plasma induced reduction of $\mathrm{TiO}_{2}$ enhances the chemical activity of surface and dissociative adsorption of water molecules on the hydrophilic surface leading to the increased hydroxyl group density. It can be concluded that fast $\mathrm{H}^{+}$transients because of their high mobility can be responsible for oxygen diffusion enhancement. This phenomenon can be explained as the result of two coexisting and competing reactions of oxidation and reduction on the surface. The different plasma reduction/oxidation state on the surface can be maintained by coordinated adjustment of an intensity of plasma radiation.

\section{References}

[1] O. Carp, C.L. Huisman, A. Reller, Prog. Solid State Chem. 32, 33 (2004).

[2] M. Anpo, M. Takeuchi, J. Catal. 216, 505 (2003).

[3] A. Fujishima, K. Honda, Bull. Chem. Soc. Jpn. 44, 1148 (1971).

[4] A. Fujishima, K. Honda, Nature 238, 37 (1972).

[5] X. Chen, S.S. Mao, Chem. Rev. 107, 2891 (2007).

[6] X. Chen, L. Liu, P.Y. Yu, S.S. Mao, Science 331, 746 (2011).

[7] S.R.J. Saunders, M. Monteiro, F. Rizzo, Prog. Mater. Sci. 53, 775 (2008).

[8] P. Perez, Corros. Sci. 49, 1172 (2007).

[9] A. Bouzoubaa, A. Markovits, M. Calatayud, C. Minot, Surf. Sci. 583, 107 (2005).

[10] S. Huet, T. Belmonte, J.M. Thiebaut, S. Bockel, H. Michel, Thin Solid Films 475, 63 (2005).

[11] W. Zhang, D.R. Sadedin, M.A. Reuter, J.C. McCallum, Mater. Forum 31, 76 (2007).

[12] L.L. Pranevicius, D. Milcius, S. Tuckute, K. Gedvilas, Appl. Surf. Sci. 258, 8619 (2012).

[13] U. Diebold, Surf. Sci. Rep. 48, 53 (2003).

[14] T.H. Quach-Kamimura, D. David, G. Beranger, A. Falanga, G. Lozes, J. Less-Common Met. 125, 59 (1986). 